\title{
TSH suppressive therapy: An overview of long-term clinical consequences
}

\author{
David S. Cooper
}

Division of Endocrinology and Metabolism, the Johns Hopkins University School of Medicine, Baltimore, U.S.A.

\begin{abstract}
An overview of long-term clinical consequences of TSH suppressive therapy provides evidence that serum TSH levels should be maintained at levels $<0.1 \mathrm{mU} / \mathrm{l}$ in high-risk (stage III and IV) patients, whereas lower-risk patients should be allowed to have their TSH levels rise into the normal range after residual disease has been ruled out by imaging and/or stimulated thyroglobulin measurement. The proper management of patients with known residual thyroid cancer, who are also elderly or who have underlying serious comorbidities (e.g., osteoporosis, cardiovascular disease, diabetes), is uncertain and requires a high degree of clinical experience and judgment.
\end{abstract}

Key words: Thyroid cancer, TSH suppression, Thyroxin therapy

Thyroid hormone treatment has been applied to prevent differentiated thyroid cancer growth or spread for almost 75 years. ${ }^{1}$ The concept behind thyrotropin (TSH) suppression therapy for differentiated thyroid cancer is that TSH is a growth factor for thyroid cancer cells. However, recent studies have questioned the rationale for TSH suppression due to a number of intriguing findings. These include the fact that many thyroid cancers lack normal functioning TSH receptors and that TSH does not necessarily regulate thyroid growth per se but more likely thyroid cellular differentiation. ${ }^{2}$ Thus, while the benefits of thyroid

\footnotetext{
Address for correspondence:

David S. Cooper, M.D., Division of Endocrinology and Metabolism, the Johns Hopkins University School of Medicine, Baltimore, MD 21287, Tel.: 410502 4926, Fax: 410955 8172, e-mail: dscooper@jhmi.edu Received 30-04-09, Revised 10-09-09, Accepted 20-10-09
}

hormone are not disputed, the actual mechanism(s) by which thyroid hormone exerts its beneficial effects may need to be reconsidered.

About $10 \%$ of patients with differentiated thyroid cancer will have a recurrence and a small but finite number will even succumb to their disease. ${ }^{3}$ The current key question for clinicians is which patients require full suppression of serum TSH levels, taking into account the risks in patients of advanced age with possible comorbidities such as underlying cardiovascular disease, and which patients do not. We must also acknowledge that low-risk patients, who make up $80 \%$ of all thyroid cancer patients, will derive little or no benefit from maintaining suppressed serum TSH levels.

Does TSH suppression actually work? A retrospective study by Pujol et $\mathrm{al}^{4}$ suggested that a suppressed 
serum TSH to undetectable levels was associated with an increased relapse-free survival in patients with differentiated thyroid cancer. On the other hand, a study by Cooper et $\mathrm{al}^{5}$ of a larger cohort of thyroid cancer patients (the National Thyroid Cancer Cooperative Study Group Registry) failed to demonstrate any effect on patients with stage I or stage II disease. Even in patients with stage III and stage IV disease the effects of TSH suppression were marginal when radioiodine therapy was included in a multivariate model. A meta-analysis of 10 studies, ${ }^{6}$ including the studies by Pujol and Cooper, did show an overall benefit from TSH suppression (relative risk of an adverse event $0.73, p<0.05$ ). However, the studies included in this meta-analysis suffer from a number of drawbacks, including possible selection bias, using thyroid hormone therapy as a surrogate for total thyroidectomy, secular bias in treatment and monitoring (for example, using serum thyroglobulin to detect recurrence in more recent studies) and, finally, the fact that many studies did not distinguish between thyroid hormone replacement therapy and suppression therapy.

Recently, there have been two more methodologically sound studies that also suggest a positive effect of TSH suppression therapy. In another study from the National Thyroid Cancer Cooperative Study Group Registry, among 1548 patients with sufficient follow-up and TSH data, TSH suppression improved overall survival in stage II patients with subnormal TSH versus normal or elevated serum TSH. ${ }^{7}$ In higher stage patients (stages III and IV), overall survival and disease specific survival were improved in patients who maintained subnormal to undetectable serum TSH levels. Limitations of this study include the fact that it was not randomized and that it is based on heterogeneous management approaches and surveillance techniques among 14 different participating institutions. In another study from a single institution in the Netherlands, ${ }^{8} 366$ patients were treated with a standard protocol with total thyroidectomy and radio-iodine therapy and followed for a mean of 8.8 years. In individual patients, the percent of serum TSH values $>4.5 \mathrm{mU} / \mathrm{l}$ was an independent predictor of death. Furthermore, serum TSH levels $>2 \mathrm{mU} / \mathrm{l}$ were also associated with thyroid cancer death and recurrence for patients with T1-3, M0 tumors.

Based on the total evidence available through 2008, the American Thyroid Association (ATA) recommended serum TSH levels $<0.1 \mathrm{mU} / 1$ for high-risk patients and 0.1-0.5 mU/l for lower-risk patients at the time of initial management. ${ }^{9}$ The European Thyroid Association (ETA) made similar recommendations. ${ }^{10}$ These groups also made recommendations for TSH suppression in the long term, after initial surgery and radioiodine therapy, and subsequent determination of whether the patient is disease free or has persistent disease. The specific recommendations of the ATA and the ETA are shown in Table 1. Briefly, both groups recommend continued suppression of serum TSH in patients who have known persistent disease and normalization of serum TSH in low-risk patients who are disease free. The two groups diverge when it comes to high-risk patients who are disease free: the ATA recommends TSH levels between 0.1 and $0.5 \mathrm{mU} / 1$, while the ETA recommends TSH levels be maintained at levels $<0.1 \mathrm{mU} / \mathrm{l}$. Neither group made any recommendations that would take into account underlying comorbidities that might be present in a thyroid cancer patient.

The reasons for recommending a normal or slightly subnormal serum TSH in groups without residual disease are the known risks of iatrogenic overt or subclinical hyperthyroidism. ${ }^{11}$ These include osteoporosis or osteopenia in postmenopausal women as well as atrial fibrillation in individuals over age 60.12 Therefore, in deciding upon the appropriate

Table 1. Recommendations of the American Thyroid Association (ATA) and the European Thyroid Association (ETA) for long-term Thyroid Stimulating Hormone suppressive therapy for differentiated thyroid cancer

\begin{tabular}{lcc}
\hline & ATA & ETA \\
\hline Persistent disease including detectable serum Tg & $<0.1 \mathrm{mU} / 1$ & $\leq 0.1 \mathrm{mU} / 1$ \\
High risk with no evidence of disease & $0.1-0.5 \mathrm{mU} / 1 \mathrm{for} 5-10$ years & $\leq 0.1 \mathrm{mU} / 1 \mathrm{for} 3-5 \mathrm{years}$ \\
Low-risk patients with apparent cure on follow-up testing & $0.3-2 \mathrm{mU} / 1$ & $0.5-1 \mathrm{mU} / 1$ \\
\hline
\end{tabular}


degree of suppression of serum TSH for an individual patient, the clinician needs to take into account the clinical stage of the patient's thyroid cancer as well as any underlying comorbidities and the patient's age before arriving at the appropriate degree of TSH suppression. This difficulty is illustrated in Figure 1. Clearly, low-risk patients with low or high risk from thyroid hormone suppression should not have their
TSH suppressed, whereas high-risk patients with low risk from suppression would benefit most from a serum TSH $<0.1 \mathrm{mU} / 1$. The difficulty arises in the high-risk cancer patient, who is apt to be older and have other comorbidities. Here, clinicians must use their best judgment, balancing risks from thyroid cancer recurrence or spread with risks of bone loss and potential cardiac rhythm disturbances.

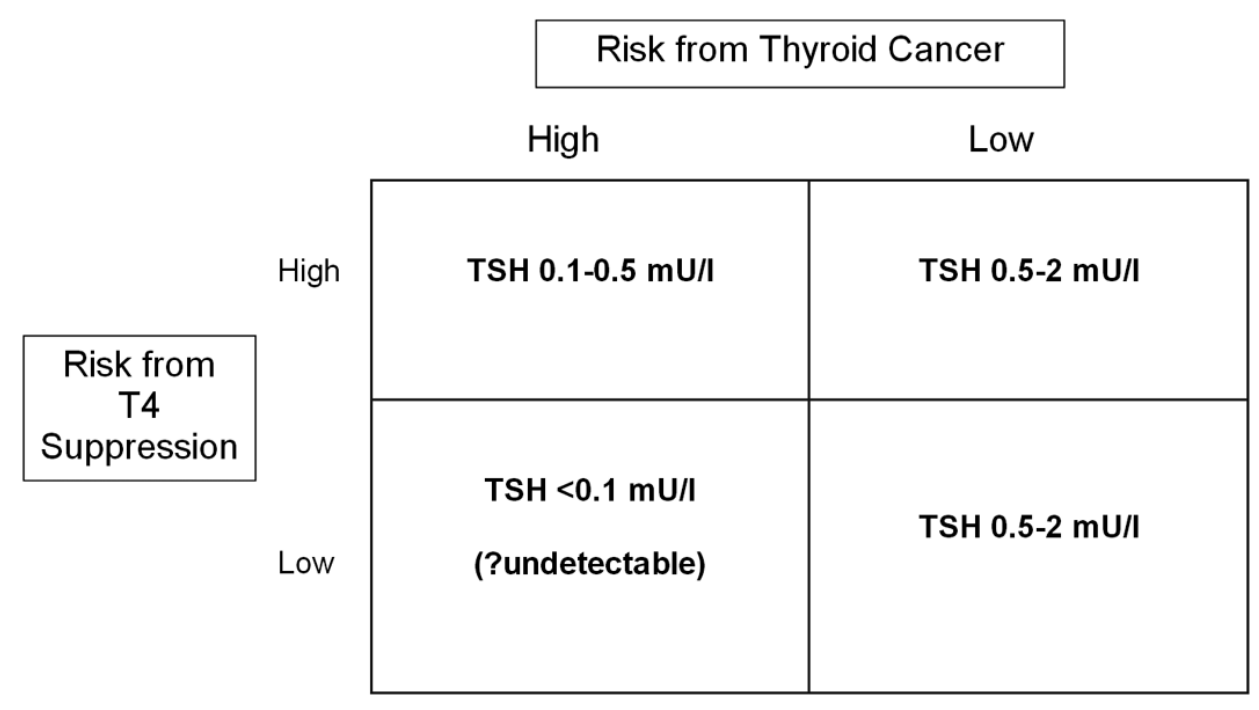

Figure 1. Stratification of TSH targets according to risk of thyroid cancer recurrence, and risk of complications from iatrogenic thyrotoxicosis.

\section{REFERENCES}

1. Dunhill TP, 1937 Surgery of the thyroid gland (The Lettsomian Lectures). BMJ 1: 460-461.

2. Brabant G, 2008 Thyrotropin suppressive therapy in thyroid carcinoma: what are the targets? J Clin Endocrinol Metab 93: 1167-1169.

3. Mazzaferri EL, Kloos RT, 2001 Clinical review 128: Current approaches to primary therapy for papillary and follicular thyroid cancer. J Clin Endocrinol Metab 86: $1447-1463$.

4. Pujol P, Daures JP, Nsakala N, Baldet L, Bringer J, Jaffiol C, 1996 Degree of thyrotropin suppression as a prognostic determinant in differentiated thyroid cancer. J Clin Endocrinol Metab 81: 4318-4323.

5. Cooper DS, Specker B, Ho M, et al, 1998 Thyrotropin suppression and disease progression in patients with differentiated thyroid cancer: results from the National Thyroid Cancer Treatment Cooperative Registry. Thyroid 8: 737-744.

6. McGriff NJ, Csako G, Gourgiotis L, Lori CG, Pucino F, Sarlis NJ, 2002 Effects of thyroid hormone suppression therapy on adverse clinical outcomes in thyroid cancer. Ann Med 34: 554-564.
7. Jonklaas J, Sarlis NJ, Litofsky D, et al, 2006 Outcomes of patients with differentiated thyroid carcinoma following initial therapy. Thyroid 16: 1229-1242.

8. Hovens GC, Stokkel MP, Kievit J, et al, 2007 Associations of serum thyrotropin concentrations with recurrence and death in differentiated thyroid cancer. J Clin Endocrinol Metab 92: 2610-2615.

9. Cooper DS, Doherty GM, Haugen BR, et al, The Revised American Thyroid Association Guidelines Taskforce, 2009 Management guidelines for patients with thyroid nodules and differentiated thyroid cancer. Thyroid 19: 1167-1214.

10. Pacini F, Schlumberger M, Dralle H, et al, European Thyroid Cancer Taskforce, 2006 European consensus for the management of patients with differentiated thyroid carcinoma of the follicular epithelium. Eur J Endocrinol 154: 787-803.

11. Biondi B, Cooper DS, 2008 The clinical significance of subclinical thyroid dysfunction Endocr Rev 29: 76131.

12. Biondi B, Filetti S, Schlumberger M, 2005 Thyroidhormone therapy and thyroid cancer: a reassessment. Nat Clin Pract Endocrinol Metab 1: 32-40. 